\title{
Correction: Prevalence of hydroxychloroquine retinopathy using 2018 Royal College of Ophthalmologists diagnostic criteria
}

Elena Marshall · Matt Robertson · Satu Kam • Alison Penwarden · Paraskevi Riga - Nigel Davies

Published online: 20 July 2020

(c) The Author(s), under exclusive licence to The Royal College of Ophthalmologists 2020

Correction to: Eye

https://doi.org/10.1038/s41433-020-1038-2
After we published this Article, we realised that data was missing from Table 3 due to a typesetting error. Table 3 has been now been corrected in the original Article (the change will appear immediately alongside this notice). 